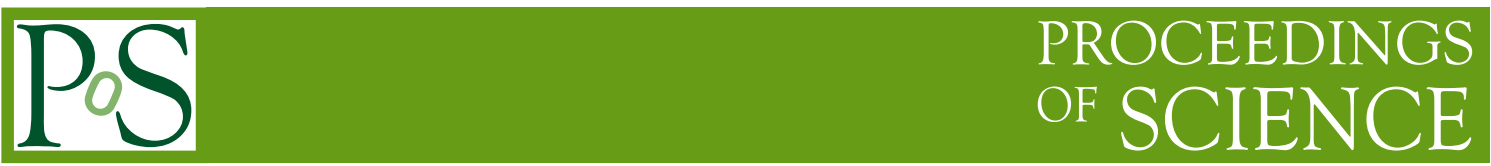

\title{
Fermi Observations of the Bright LAT GRB 160625B
}

\author{
Feraol F. Dirirsa* \\ Department of Physics, University of Johannesburg, Auckland Park 2006, South Africa \\ E-mail: fdirirsa@uj.ac.za
}

on behalf of the Fermi-LAT Collaboration

GRB 160625B was detected by both the Fermi Gamma-Ray Burst Monitor (GBM) and Large Area Telescope (LAT) instruments on board the Fermi Gamma-Ray Space Telescope, launched in June 2008. This event is associated with more than 300 high-energy photons above $100 \mathrm{MeV}$ with probabilities larger than $90 \%$. The highest energy photon is detected at 346.2 seconds after the Gamma-ray Burst Monitor (GBM) trigger with an energy of $15.3 \mathrm{GeV}$. The time-resolved spectral analysis of bursts has been presented using both the GBM and LAT data. At early times 2.05 seconds from the LAT trigger, the spectral analysis reveals an extra black body component which provides high-temperature $\mathrm{kT}=92.03 \pm 3.31 \mathrm{keV}$. The highest peak energy measured during this emission episode is $2201.0 \pm 62.7 \mathrm{keV}$ with the compatible fluence $8.38 \pm 0.05 \times 10^{-5}$ erg $\mathrm{cm}^{-2}$ in the $10 \mathrm{keV}-1 \mathrm{MeV}$ energy range. The result of temporal analysis for the LAT light curve coincident with the GBM and an optical afterglow of the Pi of the Sky has also been presented.

4th Annual Conference on High Energy Astrophysics in Southern Africa

25-27 August, 2016

South African Astronomical Observatory (SAAO), Cape Town, South Africa

${ }^{*}$ Speaker. 


\section{Introduction}

Gamma-Ray Bursts (GRBs) are the most powerful electromagnetic events in the universe, but most observations are limited to photons below a few MeV. High-energy emission above $100 \mathrm{MeV}$ from GRBs was first observed by the Energetic Gamma-Ray Experiment Telescope (EGRET, covering the energy range from $30 \mathrm{MeV}$ to $30 \mathrm{GeV}$ ) onboard the Compton Gamma-Ray Observatory (CGRO) satellite [1]. After 2008, the Fermi Gamma-Ray Space Telescope has increased the opportunity to study high-energy emission from GRBs by providing large aperture and wide field of view instruments. Two onboard instruments, the GBM [2] and the LAT [3], overlap energy bands to span from $8 \mathrm{keV}$ to above $300 \mathrm{GeV}$. The GBM consists of 12 Sodium Iodide (NaI) detectors covering energies between $8 \mathrm{keV}$ and $1 \mathrm{MeV}$. GBM also includes two Bismuth Germanate (BGO) detectors (b0, b1) covering energies between $200 \mathrm{keV}$ and $40 \mathrm{MeV}$, located on opposite sides of the spacecraft. The LAT is a pair-conversion telescope sensitive to photons with energies from $20 \mathrm{MeV}$ to $>300 \mathrm{GeV}$ [3]. When a photon enters the LAT, it is converted into an electron-positron pair after interacting in the conversion tungsten foils located between the tracker silicon strip detector planes. Since the start of science operations in early August 2008, the LAT has detected 123 GRBs with high-energy photon emission. These events, including 113 long-duration and 10 short-duration bursts seems randomly distributed in the sky map as shown in Figure 1.

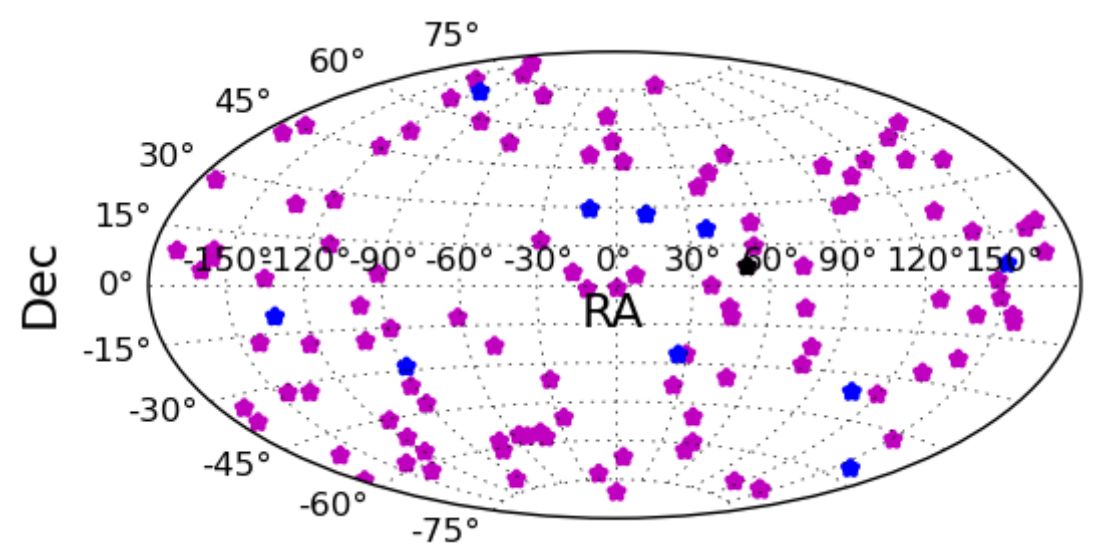

Figure 1: Distribution of the Fermi-LAT GRBs on the sky according to their best localizations on the ground. The blue and magenta star symbols correspond to short (duration $<2$ s) and long (duration $>2 \mathrm{~s}$ ) bursts, respectively. The black star is the long GRB $160625 \mathrm{~B}$.

To model the majority of GRB prompt emission spectra, an empirical model, a Band function has been used since it was first proposed in 1993 [4]. However, recent observations with the Fermi revealed deviations from the Band function (Band), sometimes in the form of an additional blackbody (BB) thermal component [5], while on other occasions in the form of an additional power law (PL) component extending to high energies. In some bursts, a high energy cut-off in the MeV regime [6] and an additional PL component [7] that model the lower energy was observed. In this paper, we present the spectral analysis of GRB 160625B by performing a Band function, a Band function with an extra black body (Band+BB) and Band function with two an additional extra component of black body and power law (Band+BB+PL) models. 


\section{Observations}

At 22:40:16.28 UT on June 25, 2016 (hereafter $\mathrm{T}_{0}$ ), the GBM triggered on and located the bright burst GRB 160625B with the trigger number 160625945 and triggered again the same GRB at 22:51:16.03 UT with the trigger number 160625952 [8], with emission evident between triggers, including the time at which the Fermi-LAT triggered. The burst was within the LAT field-of-view initially at an angle of $42^{\circ}$ from the boresight at the time of GBM trigger. The GBM light curve consists of multiple peaks over the duration of $\mathrm{T}_{90}=460 \mathrm{~s}$ [8].

At 22:43:24.82 UT the Fermi-LAT triggered on a bright pulse from the GRB 160625B [9]. From the LAT data, the burst was localized at RA, Dec $(\mathrm{J} 2000)=308.3^{\circ}, 6.9^{\circ}$ with an error radius of $0.5^{\circ}$ ( $90 \%$ containment, systematic error only), enabling Target of Opportunity (ToO) observations to begin $\sim 24 \mathrm{hr}$ after the trigger with the narrow field instruments on Swift. The number of LAT observed events are $>300$ photon candidates above $100 \mathrm{MeV}$, which is comparable to that of the other bright LAT bursts, GRB 080916C, GRB 090510 and GRB 090902B. The LAT emission became detectable and in coincidence with the second spiky bright pulse observed by GBM; however, a series of emission photon with high energy are clearly visible in the light curve just after $\mathrm{T}_{0}$ as shown in Figure 2a, but not sufficient to alert the LAT. The most energetic photon was observed 346.2 seconds after $\mathrm{T}_{0}$, had an energy of $\mathrm{E}=15.3 \mathrm{GeV}$ and was associated to the GRB with a high (0.99 using the gtsrcprob Fermi Science tool) probability. The best LAT on-ground localization is RA, Dec $=308.56^{\circ}, 6.93^{\circ}(\mathrm{J} 2000)$, with an error radius of $0.06^{\circ}(90 \%$ containment, statistical error only) and fully compatible with the position of the afterglow detected by Swift/XRT [10].

Indeed, based upon the GCN report issued for the LAT detection, a Swift ToO observation was performed, and the Swift-XRT an afterglow for GRB 160625B was detected with $9.6 \mathrm{ks}$ and localized at $(\mathrm{RA}, \mathrm{Dec})=308.5969^{\circ}, 6.9196^{\circ}$ ) with an uncertainty of $3.8^{\circ}$ (radius, $90 \%$ confidence) [10] . This is $19.7^{\circ}$ from the LAT position. VLT observations determined a redshift for GRB 160625B of $z=1.406$, using the X-shooter spectrograph [11], and the TNG equipped with DOLORES and the LR-B grism also confirmed a redshift of $z=1.41 \pm 0.01$ for this burst. Cameras of the Pi of the Sky North observatory observed optical emission of the GRB 160625B 48s after Fermi GBM trigger and 140 seconds before the LAT 488587408 trigger [12]. Both cameras, 35 and 39, identified a new object on exposures starting just before the time of the trigger. Mini-MegaTORTORA nine-channel wide-field monitoring system observed [13] a bright optical flash of GRB 160625B, on a frame coincident with LAT trigger time $\left(\sim \mathrm{T}_{0}+173.7\right.$ to $\left.\sim \mathrm{T}_{0}+201.8\right)$ at position reported by Troja et al. [14], with the magnitude of about $\mathrm{V}=8.8 \mathrm{mag}$. The system started observing its error box at $\mathrm{T}_{0}+52$ seconds after GBM and 136 seconds before the LAT trigger. The bright optical counterpart of this source is more consistent with GRB 120711A, which was also observed by several ground-based telescopes that detected a powerful optical flash peaking at an R-band brightness of $~ 11.5$ mag [15]. RATIR Optical/NIR [14] and MITSuME/Ishigakijima Optical [16] also detected an optical afterglow emission.

\subsection{Light Curves}

The NaI, BGO and LAT light curves of GRB 160625B are shown in Figure 2. Comparing to all panels in Figure 2a, we see that the first short pulse, clearly visible in the NaI (n9) light curve, are not seen in BGO and LAT data, while the second broader feature emission is in coincidence 
with the high energy count rate in the LAT data. An event with energy $0.2 \mathrm{GeV}$ was observed at 10 $\mathrm{s}$, after the first soft GBM emission has ended, consistent with a delayed arrival of the high-energy emission which is similar to other bright LAT GRBs [17].
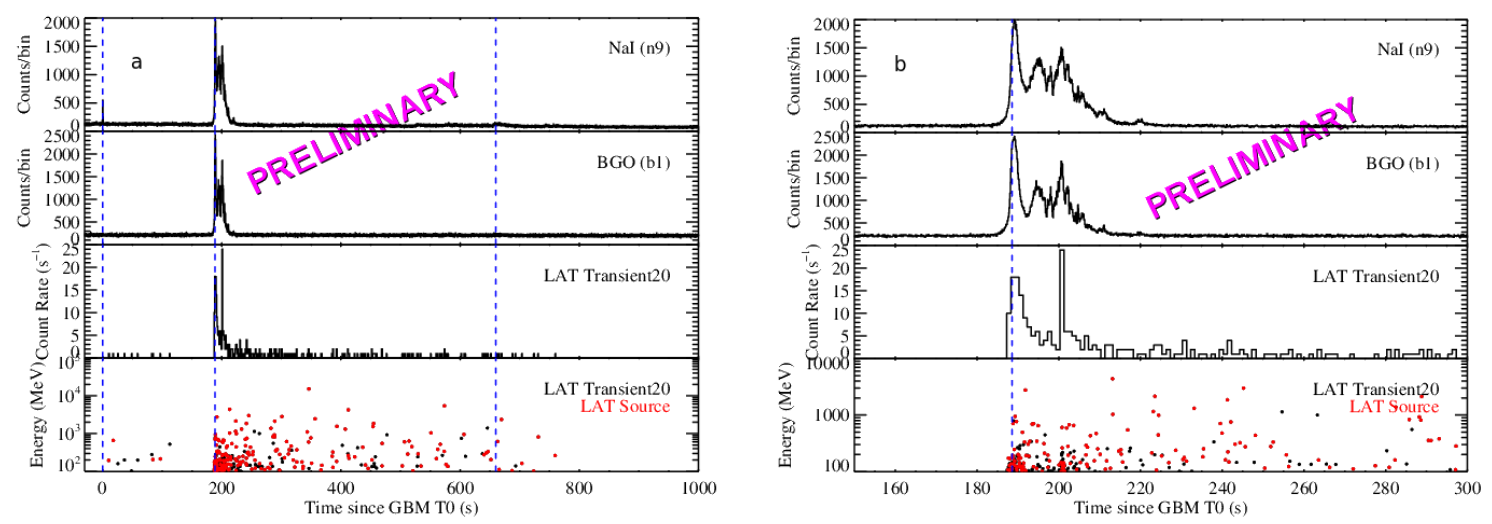

Figure 2: GBM and LAT light curves of GRB 160625B. a) the blue lines indicate, the first GBM trigger time at $488587220 \mathrm{MET}$, the LAT trigger time at $488587408 \mathrm{MET}$ and the second GBM trigger time at 488587880 MET from left to right, respectively. The top two panels show the light curve of NaI(n9) detector and of the $\mathrm{BGO}(\mathrm{b} 1)$ detector. The third panel indicates the LAT light curve for the "transient" event selection above $100 \mathrm{MeV}$ and the fourth panel shows the LAT photons which passed the "transient" and "source" event selection above $100 \mathrm{MeV}$. b) Zoom on the spiky light curve for which spectral analysis has been performed. A blue line indicates the LAT trigger time.

\section{Spectral Analyses}

\subsection{Data Selection}

We use the data from the GBM NaI (n9) detector that have source angles less than $50^{\circ}$, the data of BGO detector (b1) closer to the source and the LAT. The spectral analysis of GRB 160625B is performed for the second broad emission part from $\sim \mathrm{T}_{0}+187.43$ to $\sim \mathrm{T}_{0}+212.00$ seconds, around the spiky light curve of both the LAT and GBM events, (see Figure 2b) with the software package Rmfit (version 4.3.2) using Castor Statistics (C-Stat) [18]. In order to subtract the GBM background spectrum for the spectral analysis, we defined two background intervals before and after the prompt emission for every energy channel. For each emission episode, we fitted with a second-order polynomial function to a user defined background and interpolated this fit across the source interval. We used the standard 128 energy bins of the CSPEC data-type, for NaI using the channels from $\sim 8 \mathrm{keV}$ to $\sim 905 \mathrm{keV}$ cutting out the over-flow high-energy channels as well as (corresponding to the Iodine K-edge from $\sim 30-\sim 40 \mathrm{keV}$, see [2]). For BGO (b1), we used data from $\sim 220 \mathrm{keV}$ to $\sim 9.5 \mathrm{MeV}$. For the analysis of LAT observations, the pass 8 Transient class events (Transient20E) used for the event selection with a $10^{\circ}$ radius of interest. The data is binned in 30 logarithmically spaced energy steps between $100 \mathrm{MeV}$ and $300 \mathrm{GeV}$. We derive the observed spectrum and the detector response matrix using the Fermi Science Tools gtbin and gtrspgen, respectively. To produce a background spectrum file, the background-estimation tool so-called gtbkg was used. 


\subsection{Joint LAT-GBM Spectral Analysis}

The soft peak precursor episode that initially triggered GBM was about 1 second long from $\mathrm{T}_{0}$ as shown in Figure 2a and well fit by compotonized model with peak energy $\mathrm{E}_{\text {peak }}=69.9 \pm 1.5$ $\mathrm{keV}$ and power law index is $-0.27 \pm 0.07$. The energy flux $(10-1000 \mathrm{keV})$ in this time interval is $1.53 \pm 0.03 \times 10^{-6} \mathrm{erg} \mathrm{cm}^{-2} \mathrm{~s}^{-1}$.

We have also performed time-resolved spectral analysis of the combined GBM and LAT data over a period corresponding to the most intense part of the prompt emission (i.e., $\mathrm{T}_{0}+187.43-\mathrm{T}_{0}$ $+212.00 \mathrm{~s}$ ), where $158 \mathrm{LAT}$ photons above $100 \mathrm{MeV}$ with probability greater than $90 \%$ of being associated with the burst are detected. For spectral analysis, different empirical models are considered: a smoothly broken power law, a Band function (Band) [4] with peak energy $\left(\mathrm{E}_{\text {peak }}\right)$ which smoothly connects two power laws, a Band function with an additional power law (Band+PL) and Band function with black body (Band+BB) component. Results of our combined GBM and LAT time-resolved spectral analysis are given in Table 1.
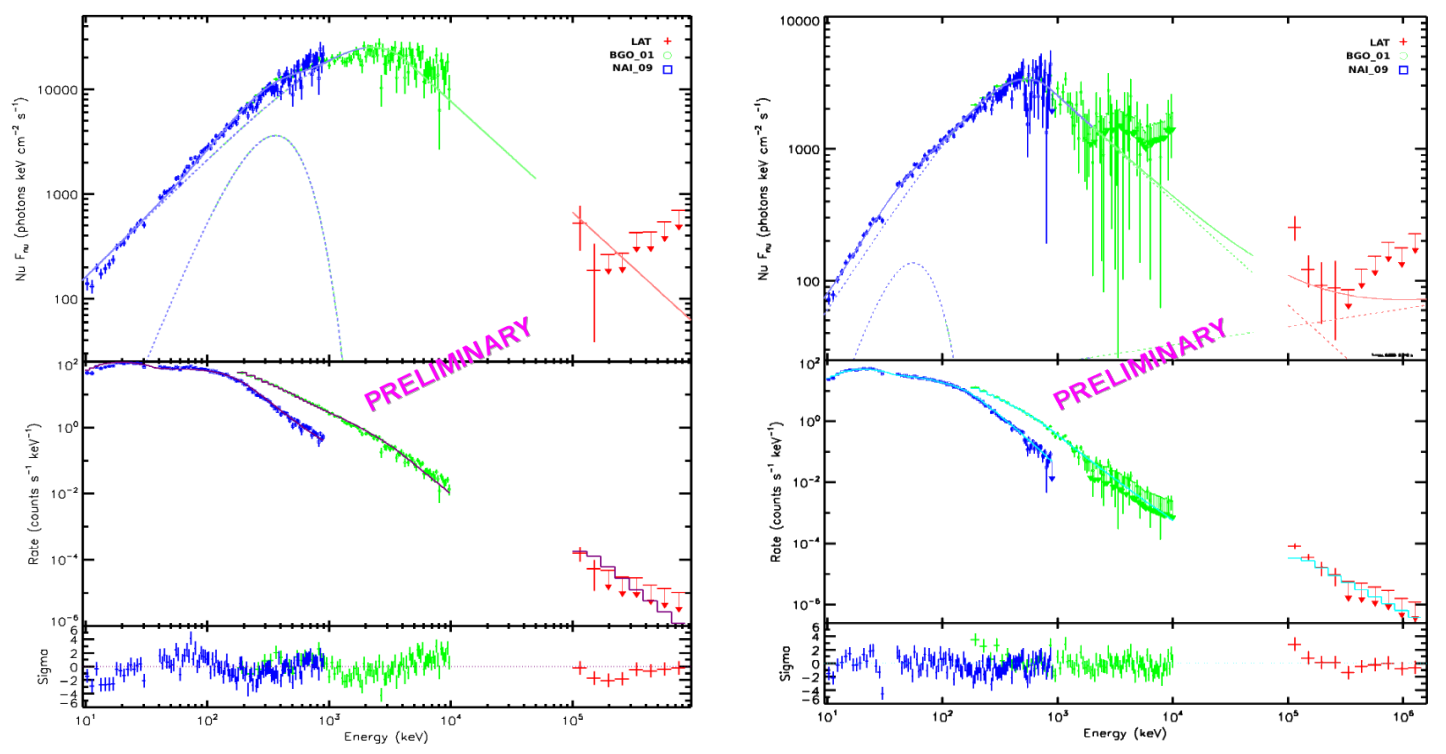

Figure 3: Results of the spectral fit of the first time bin, $\mathrm{T}_{0}+187.43-\mathrm{T}_{0}+189.48 \mathrm{~s}$, using a Band function with an additional black body function (left) and the fifth time bin, $\mathrm{T}_{0}+201.76-\mathrm{T}_{0}+212.00$, performing a Band function with an additional two components black body and power law functions (right). Top panels show counts spectrum with folded model and the bottom panels indicate the counts spectrum with folded model and residuals.

At early times (first $2.05 \mathrm{~s}$ ), adding thermal component to a Band model provide the temperature $\mathrm{kT}=92.03 \pm 3.31 \mathrm{keV}$ while the peak energy is shifted from $1441 \pm 30.2 \mathrm{keV}$ to $2201 \pm 62.7$ $\mathrm{keV}$. This improves the fit about 178 in $\Delta \mathrm{C}$-Stat. This fit is shown in Figure 3 (left). Moreover, fine time-resolved spectral analysis may constrain the emission of this burst, since there is a continuous change in shape of spectra with time evolution.

We analyzed the time-resolved spectra of GRB 160625B. This procedure divides the bursts into 5 time intervals which are indicated in Table 1. We fitted all the intervals from (a) to (e) by 
Band function and the spectral evolution is evident in this function component from changing $\mathrm{E}_{\text {peak }}$ values throughout the burst but the fits are not well constraint the emission. The addition of a black body component to the Band model improves the fit from interval (a to e) significantly in $\Delta \mathrm{C}$-Stat (corresponding to $>5 \sigma$ significance). The peak energy $\mathrm{E}_{\text {peak }}$ shifted to high values from (a) to (c) interval, while the power law indices are harder than the Band function alone. In interval (e), the emission can be fitted by a Band function with two extra components BB and PL with photon spectral index $(\gamma)=-1.88 \pm 0.09$. This fit is shown in Figure 3 (right). An additional BB and $\mathrm{BB}+\mathrm{PL}$ an extra component provides a better fit, as its inclusion causes an improvement of 46 and 53 in $\Delta \mathrm{C}$-Stat, respectively over the Band function alone.

Table 1: The result of time-resolved spectral parameters. For the first four bins, Band+BB model constrain the emission while the fifth bin can be modelled with a Band+BB+PL.

\begin{tabular}{|c|c|c|c|c|c|c|c|c|}
\hline & Time range* & $\alpha$ & $\beta$ & $\mathrm{E}_{\text {peak }}(\mathrm{keV})$ & $\mathrm{kT}(\mathrm{keV})$ & $\gamma$ & C-Stat/dof** & Model \\
\hline \multirow[t]{2}{*}{ (a) } & $187.43-189.48$ & $-0.73 \pm 0.01$ & $-2.78 \pm 0.05$ & $1441.0 \pm 30.2$ & & & $596.2 / 203$ & Band \\
\hline & & $-0.85 \pm 0.01$ & $-3.11 \pm 0.07$ & $2201.0 \pm 62.7$ & $92.03 \pm 3.31$ & & $417.9 / 201$ & Band+BB \\
\hline \multirow[t]{2}{*}{ (b) } & $189.48-191.52$ & $-0.72 \pm 0.01$ & $-2.58 \pm 0.03$ & $789.3 \pm 18.4$ & & & $388.7 / 205$ & Band \\
\hline & & $-0.69 \pm 0.02$ & $-2.61 \pm 0.03$ & $843.0 \pm 24.0$ & $25.03 \pm 2.75$ & & $352.8 / 203$ & Band+BB \\
\hline \multirow[t]{2}{*}{ (c) } & $191.52-195.62$ & $-0.70 \pm 0.01$ & $-2.70 \pm 0.03$ & $583.9 \pm 10.2$ & & & $397.3 / 210$ & Band \\
\hline & & $-0.65 \pm 0.02$ & $-2.71 \pm 0.03$ & $594.7 \pm 12.5$ & $17.52 \pm 1.71$ & & $370.91 / 208$ & Band+BB \\
\hline \multirow[t]{2}{*}{ (d) } & $195.62-201.76$ & $-0.70 \pm 0.01$ & $-2.79 \pm 0.03$ & $715.7 \pm 9.98$ & & & $406.7 / 204$ & Band \\
\hline & & $-0.62 \pm 0.02$ & $-2.79 \pm 0.03$ & $707.3 \pm 11.3$ & $15.53 \pm 0.90$ & & $361.2 / 202$ & Band+BB \\
\hline \multirow[t]{3}{*}{ (e) } & $201.76-212.00$ & $-0.82 \pm 0.01$ & $-2.66 \pm 0.03$ & $540.3 \pm 10.7$ & & & $359.3 / 203$ & Band \\
\hline & & $-0.71 \pm 0.03$ & $-2.67 \pm 0.03$ & $540.3 \pm 12.6$ & $13.90 \pm 0.82$ & & $313.6 / 201$ & Band+BB \\
\hline & & $-0.65 \pm 0.07$ & $-2.85 \pm 0.09$ & $543.6 \pm 14.6$ & $14.89 \pm 0.95$ & $-1.88 \pm 0.09$ & $306.6 / 199$ & Band $+B B+P L$ \\
\hline
\end{tabular}

Notes. ${ }^{*}$ Measured in seconds since trigger time $\mathrm{T}_{0} . \quad \alpha$ and $\beta$ are the low and high energy spectral index for Band function. ${ }^{* *}$ the ratio of the value of the $\mathrm{C}$-stat resulting from the fit and the associated degrees of freedom (dof). This results cannot be used to estimate goodness of fit nevertheless compare to C-stat for models [18, 19].

\section{Temporally Extended High-Energy Emission}

We might understand the features of the gamma-ray bursts from a pattern emerge when the temporal structure of their emission are analyzed by considering the overall time scales of the burst. Observations of extended, high-energy emission are, however, scarce. To characterize the temporally extended high-energy emission of GRB 160625B, the beginning of the LAT data has been chosen at $187.43 \mathrm{~s}$ from $\mathrm{T}_{0}$ for energy $\mathrm{E}>100 \mathrm{MeV}$. This observation was divided in time bins, in all of them, unbinned maximum likelihood analysis was performed. We modeled the LAT photon spectrum as a power law with a spectral index [i.e., the spectrum $N(E) \propto E^{\alpha}$ ]. We found evidence of spectral evolution during the high energy emission. The temporal decay is very steep, with a photon index of $-2.35 \pm 0.13$, which is unusual. The reduced $\chi^{2}$ for this power law fit is 0.44 . The LAT flux density also fitted by a broken power law (BPL) with an initial shallow decay $\alpha_{1}=-2.23 \pm 0.12$, the time break at 205.5 is followed by rise and a steep late decay $\alpha_{1}=-7.12 \pm$ 1.73 as shown by cyan bend line in Figure 4. The PL fit of LAT flux density is softer and steeper than the BPL. In our analysis, we used also an optical afterglow data from the Pi of the Sky with two camera Id $=35$ and 39 [12]. The telescope observed at the position of GBM $40 \mathrm{~s}$ after trigger and $148 \mathrm{~s}$ before the LAT trigger of GRB 160625B. The two camera Id $=35$ and 39 light curves 


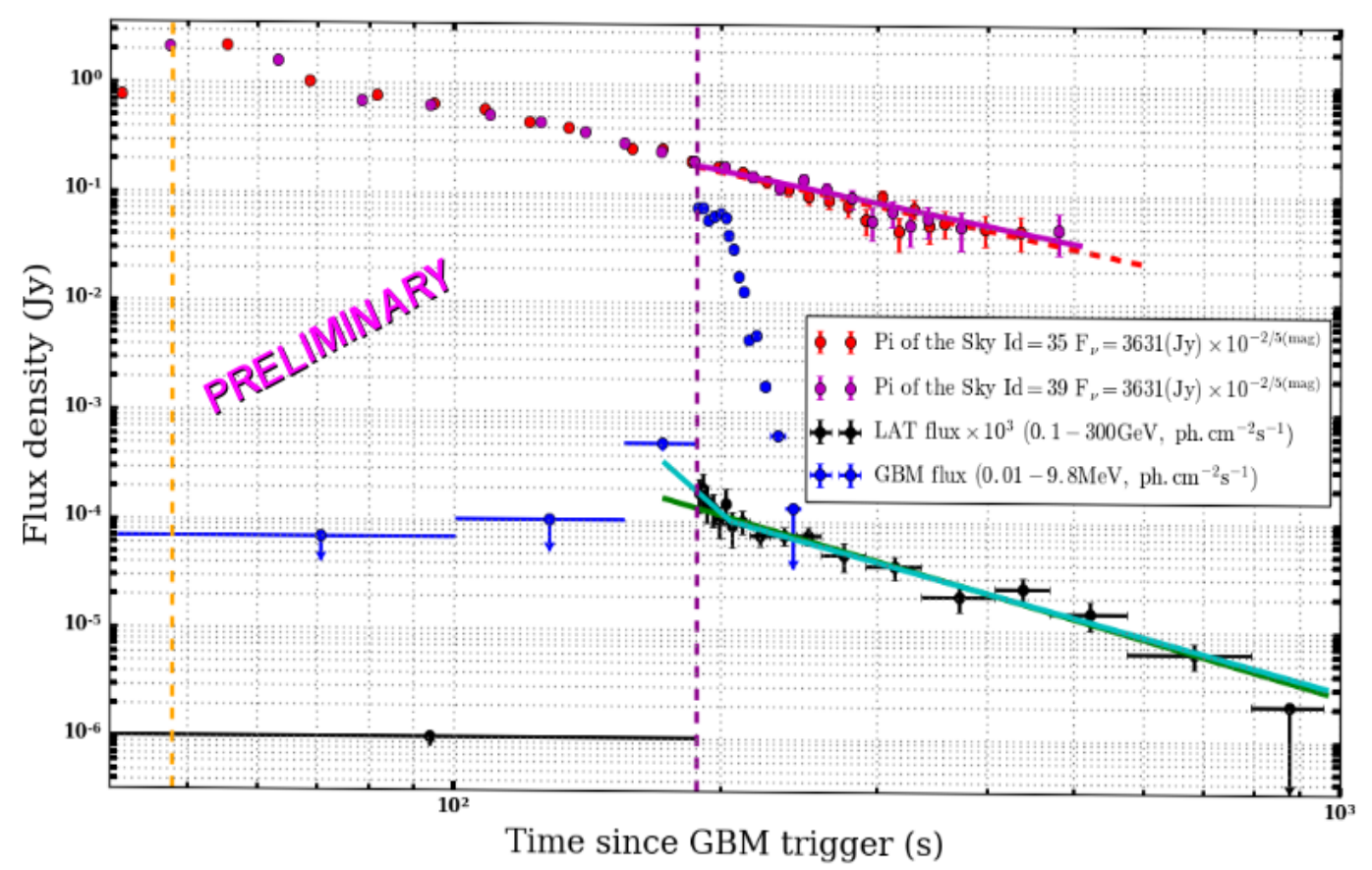

Figure 4: The fluxes observed by the Fermi-LAT/GBM and Pi of the Sky telescopes. A blue filled circles show the data of GBM. The red broken and magenta lines depict the PL fit of data of Pi of the Sky camera Id 35 and 39, respectively. The optical afterglow data is shifted by $\sim 153$ seconds from the LAT trigger towards the GBM trigger time. Afterwards the data that coincide with the LAT is fitted by a power law. A green line is a PL fit of LAT data while a cyan line is a broken power law obtained from the LAT fit.

are best fit by a power law with the data coinciding of LAT, index $-1.74 \pm 0.16$ (the reduced $\chi^{2}$ $=0.83$ ) and $-1.64 \pm 0.20$ (the reduced $\chi^{2}=0.75$ ), respectively. Their power law index are harder than the LAT.

\section{Summary}

The time-resolved spectrum of GRB 160625B are presented using both the GBM and LAT instruments on Fermi. At early times (first $2.05 \mathrm{~s}$ from the LAT trigger), the spectral analysis reveals an extra black body component which provides the temperature $\mathrm{kT}=92.03 \pm 3.31 \mathrm{keV}$. The highest peak energy measured during this emission episode is $2201.0 \pm 62.7 \mathrm{keV}$, and the corresponding fluence is $8.38 \pm 0.05 \times 10^{-5} \mathrm{erg} \mathrm{cm}^{-2}$ in the $10 \mathrm{keV}-1 \mathrm{MeV}$ energy range. The preliminary analysis showed that from the interval $(a-d)$, the time-resolved spectral analysis is modelled by a Band plus black body function, while the last interval (e) is fitted by a band function with two extra components black body and power law function. Thus, further detailed spectral analysis and additional models for comparison have to be carried out to study the spectra emission of GRB 160625B. The temperature of the BB falls exponentially similar to the $\mathrm{E}_{\text {peak }}$ except in interval (d). In the first two time intervals of spectral analysis, the spectral energy indices are less than $-2 / 3$ expected from optically thin synchrotron emission [20]. We find that GRB 160625B 
is more similar to GRB 080825C and GRB 081006A with comparable appearances of late highenergy photon emission. The time-resolved spectra of the main episode $\left(\sim \mathrm{T}_{0}+180-\sim \mathrm{T}_{0}+200\right.$ seconds) have been also done by Lü et al. [21] at different time bins. A function composing of a multi-color black body component plus a cutoff power-law component (CPL), can be fitted the spectra of the burst, however; the photon index and the cutoff energy of the CPL component does not show a clear evolution trend. The brightest peak in the LAT light curve is coincident with the GBM and an optical afterglow data from the Pi of the Sky. The LAT energy flux light curve is well described by a broken power law with the first steep index $\alpha_{1}=-7.12 \pm 1.73$, which appears to be softer than the second index $\alpha_{2}=-2.23 \pm 0.12$.

\section{Acknowledgments}

The Fermi-LAT Collaboration acknowledges support for LAT development, operation and data analyses from NASA and DOE (United States), CEA/Irfuand IN2P3/CNRS (France), ASI and INFN (Italy), MEXT, KEK, and JAXA (Japan), and the K.A. Wallenberg Foundation, the Swedish Research Council and the National Space Board (Sweden). Science analyses support in the operations phase from INAF (Italy) and CNES (France) is also gratefully acknowledged. The work presented in this paper was supported in part by an MWGR 2016 grant from the National Research Foundation with Grant No. 93273.

\section{References}

[1] C. Fichtel, D. Bertsch, J. Chiang, B. Dingus, J. Esposito, J. Fierro, R. Hartman, S. Hunter, G. Kanbach, D. Kniffen, et al., "The first energetic gamma-ray experiment telescope (egret) source catalog," The Astrophysical Journal Supplement Series, vol. 94, pp. 551-581, 1994.

[2] C. Meegan et al. The Astrophysical Journal, vol. 702, no. 1, p. 791, 2009.

[3] W. B. Atwood et al., "The large area telescope on the fermi gamma-ray space telescope mission," The Astrophysical Journal, vol. 697, no. 2, p. 1071, 2009.

[4] D. Band et al., "Batse observations of gamma-ray burst spectra. i-spectral diversity," The Astrophysical Journal, vol. 413, pp. 281-292, 1993.

[5] S. Guiriec, C. Kouveliotou, F. Daigne, B. Zhang, R. Hascoët, R. Nemmen, D. Thompson, P. Bhat, N. Gehrels, M. Gonzalez, et al., "Toward a better understanding of the grb phenomenon: a new model for grb prompt emission and its effects on the new lint-epeak, irest, nt relation," The Astrophysical Journal, vol. 807, no. 2, p. 148, 2015.

[6] M. Ackermann et al., "Constraining the high-energy emission from gamma-ray bursts with fermi," The Astrophysical Journal, vol. 754, no. 2, p. 121, 2012.

[7] A. A. Abdo et al., "Fermi observations of grb 090902b: A distinct spectral component in the prompt and delayed emission," The Astrophysical Journal Letters, vol. 706, no. 1, p. L138, 2009.

[8] E. Burns, “GRB 160625B: Fermi GBM initial observations.," GRB Coordinates Network, vol. 19581, 2016.

[9] F. Dirirsa et al., "GRB 160625B: Fermi-LAT detection of a bright burst.," GRB Coordinates Network, vol. 19580, 2016. 
[10] A. Melandri et al., "GRB 160625B: Swift-XRT afterglow detection.," GRB Coordinates Network, vol. 19585, 2016.

[11] D. Xu et al., “GRB 160625B: VLT/X-shooter redshift.,” GRB Coordinates Network, vol. 19600, 2016.

[12] T. Batsch et al., "Pi of the Sky detection of an optical flash accompanying GRB 160625B.," GRB Coordinates Network, vol. 19615, 2016.

[13] S. Karpov, G. Beskin, S. Bondar, E. Ivanov, E. Katkova, N. Orekhova, A. Perkov, A. Biryukov, and V. Sasyuk, "GRB 160625B: Mini-MegaTORTORA detection of bright optical flash simultaneous with LAT event.," GRB Coordinates Network, vol. 19603, 2016.

[14] E. Troja et al., "GRB 160625B: RATIR Optical and NIR Afterglow Detection.," GRB Coordinates Network, vol. 19588, 2016.

[15] A. Martin-Carrillo, L. Hanlon, M. Topinka, A. LaCluyzé, V. Savchenko, D. Kann, A. Trotter, S. Covino, T. Krühler, J. Greiner, et al., "Grb 120711a: an intense integral burst with long-lasting soft $\gamma$-ray emission and a powerful optical flash,” Astronomy \& Astrophysics, vol. 567, p. A84, 2014.

[16] D. Kuroda et al., "GRB 160625B: RATIR Optical and NIR Afterglow Detection.," GRB Coordinates Network, vol. 19599, 2016.

[17] M. Ackermann, M. Ajello, K. Asano, M. Axelsson, L. Baldini, J. Ballet, G. Barbiellini, M. Baring, D. Bastieri, K. Bechtol, et al., "Detection of a spectral break in the extra hard component of grb 090926a," The Astrophysical Journal, vol. 729, no. 2, p. 114, 2011.

[18] W. Cash, "Parameter estimation in astronomy through application of the likelihood ratio," The Astrophysical Journal, vol. 228, pp. 939-947, 1979.

[19] H.-F. Yu, R. D. Preece, J. Greiner, P. N. Bhat, E. Bissaldi, M. S. Briggs, W. H. Cleveland, V. Connaughton, A. Goldstein, A. Von Kienlin, et al., "The fermi gbm gamma-ray burst time-resolved spectral catalog: brightest bursts in the first four years," Astronomy \& Astrophysics, vol. 588, p. A135, 2016.

[20] F. Ryde and A. Pe'er, "Quasi-blackbody component and radiative efficiency of the prompt emission of gamma-ray bursts," The Astrophysical Journal, vol. 702, no. 2, p. 1211, 2009.

[21] H.-J. Lü, J. Lü, S.-Q. Zhong, X.-L. Huang, H.-M. Zhang, W. Xie, R.-J. Lu, and E.-W. Liang, "Extremely bright GRB 160625B with multi-episodes emission: Evidences for long-term ejecta evolution," ArXiv e-prints, Feb. 2017. 